\title{
Glycine decarboxylase is a target for transcriptional repressor Snail
}

\author{
Guohua Chen', Stephanie Lucas², Jian Wang ${ }^{1 *}$ \\ From Metabolism, Diet and Disease 2014: Cancer and metabolism \\ Washington DC, USA. 28-30 May 2014
}

\begin{abstract}
Background
Aberrant glycine metabolism, an emerging hallmark of cancer, contributes to the aggressive proliferation [1] and invasion [2] of tumor cells. Glycine decarboxylase (GLDC) is the rate-limiting enzyme of glycine cleavage system that catabolizes glycine to feed one-carbon metabolism in mitochondria. A recent study suggests it plays a crucial role in tumorigenesis by promoting proliferation and pyrimidine synthesis of tumor initiation cells [3]. However, while its tumorigenic role is being unveiled, how it is de-regulated in tumor cells remains obscure. Here we present results showing that GLDC is a direct target for Snail, the key transcriptional repressor controlling the epithelial-mesenchymal transition (EMT) program during embryogenesis and tumor progression.
\end{abstract}

\section{Materials and method}

We have generated clones of lung cancer A549 cells expressing Snail under the control of doxycyclineinducible promoter. Genes that are significantly down regulated in Snail-expressing cells are identified by comparative gene expression profiling with Illumina RNA-seq.

\section{Results}

DAVID Gene Ontology analysis of Snail-responsive genes shows highest enrichment for cell adhesion, extracellular matrix, cytoskeleton, cell migration and proliferation, which validates the EMT functionality of transfected Snail. Interestingly, GLDC is found among the genes whose expressions are most down regulated in Snail-expressing cells. We further show that GLDC expression is significantly repressed at both mRNA and protein levels in response to Snail induction or by TGF- $\beta$ treatment in a dose- and time-dependent manner in multiple cell lines.
Sequence analysis reveals a couple of evolutionally conserved putative Snail-binding elements existing at the upstream proximal to the transcription start site within GLDC promoter. Using luciferase reporter assay and ChIP analysis, we show that wide-type not mutant Snail represses GLDC promoter activity by binding to GLDC promoter. Thus our genome-wide transcriptome screening and molecular characterization reveal GDLC as a new direct target for Snail. Given the critical role of Snail in endowing migratory and invasive cellular phenotypes, these results suggest the potential contribution of GLDCmediated cellular metabolism to metastatic tumor progression.

\section{Acknowledgements}

This study is partly supported by WSU CVRI Isis award to JW.

\section{Authors' details}

'Department of Pathology, Wayne State University, Detroit, MI 48201, USA.

${ }^{2}$ Department of Oncology, Detroit, MI 48201, USA.

Published: 28 May 2014

\section{References}

1. Jain M, Nilsson R, Sharma $S$, et al: Metabolite profiling identifies a key role for glycine in rapid cancer cell proliferation. Science 2012, 336:1040-1044.

2. Sreekumar A, Poisson LM, Rajendiran TM, et al: Metabolomic profiles delineate potential role for sarcosine in prostate cancer progression. Nature 2009, 457:910-914.

3. Zhang WC, Shyh-Chang N, Yang H, et al: Glycine decarboxylase activity drives non-small cell lung cancer tumor-initiating cells and tumorigenesis. Cell 2012, 148:259-272.

\section{doi:10.1186/2049-3002-2-S1-P83}

Cite this article as: Chen et al:: Glycine decarboxylase is a target for transcriptional repressor Snail. Cancer \& Metabolism 2014 2(Suppl 1):P83. 